Supporting Information for

\title{
Nature of $\equiv \mathrm{SiOCrO}_{2} \mathrm{Cl}$ and $(\equiv \mathrm{SiO})_{2} \mathrm{CrO}_{2}$ Sites
}

\section{Prepared by Grafting $\mathrm{CrO}_{2} \mathrm{Cl}_{2}$ onto Silica}

Cori A. Demmelmaier, ${ }^{a}$ Rosemary E. White, ${ }^{a}$ Jeroen A. van Bokhoven, ${ }^{c}$ and Susannah L. Scott ${ }^{a, b *}$

\section{Table of contents}

Page

Figure S1 Single-scattering refinement of the $\equiv \mathrm{SiOCrO}_{2} \mathrm{Cl}$ model to EXAFS of $\mathrm{CrO}_{2} \mathrm{Cl}_{2}$-modified S952-450

Table S1 Fit parameters for single-scattering refinement of the $\equiv \mathrm{SiOCrO}_{2} \mathrm{Cl}$ model to EXAFS of $\mathrm{CrO}_{2} \mathrm{Cl}_{2}$-modified S952-450

Figure S2 Single-scattering refinement of the $\equiv \mathrm{SiOCrO}_{2} \mathrm{Cl}$ model to EXAFS of $\mathrm{CrO}_{2} \mathrm{Cl}_{2}$-modified S952-800

Table S2 Fit parameters for single-scattering refinement of the $\equiv \mathrm{SiOCrO}_{2} \mathrm{Cl}$ model to EXAFS of $\mathrm{CrO}_{2} \mathrm{Cl}_{2}$-modified S952-800

Table S3 Cartesian Coordinates for Optimized Structure of $\mathbf{I}$

Table S4 Cartesian Coordinates for Optimized Structure of Ia 


\section{Table of contents, continued}

Table S6 Cartesian Coordinates for Optimized Structure of Ic S10

Table S7 Cartesian Coordinates for Optimized Structure of II $\quad$ S11

Table S8 Cartesian Coordinates for Optimized Structure of IIa $\quad$ S12

Table S9 Cartesian Coordinates for Optimized Structure of IIIb S13

Table S10 Cartesian Coordinates for Optimized Structure of IIc $\quad$ S14

Table S11 Cartesian Coordinates for Optimized Structure of III S15

Table S12 Cartesian Coordinates for Optimized Structure of IV S16

$\begin{array}{lll}\text { Table S13 Cartesian Coordinates for Optimized Structure of V } & \text { S17 }\end{array}$

Table S14 Cartesian Coordinates for Optimized Structure of VI $\quad$ S18

Table S15 Cartesian Coordinates for Optimized Structure of $\mathrm{CrO}_{2} \mathrm{Cl}_{2} \quad \mathrm{~S} 19$

Table S16 Cartesian Coordinates for Optimized Structure of $\mathrm{HCl} \quad$ S20

Table S17 Cartesian Coordinates for Optimized Structure of $\mathrm{H}_{2} \mathrm{O} \quad$ S20 
Figure S1. Single-scattering refinement of the $\equiv \mathrm{SiOCrO}_{2} \mathrm{Cl}$ model to EXAFS of $\mathrm{CrO}_{2} \mathrm{Cl}_{2}$ modified S952-450: (a) in $\mathrm{k}^{3}$-weighted k-space (red points and line); and (b) in non-phasecorrected R-space (imaginary, black points; FT magnitude, red points). Parameters for the curvefit (blue lines) are given in Table S1.
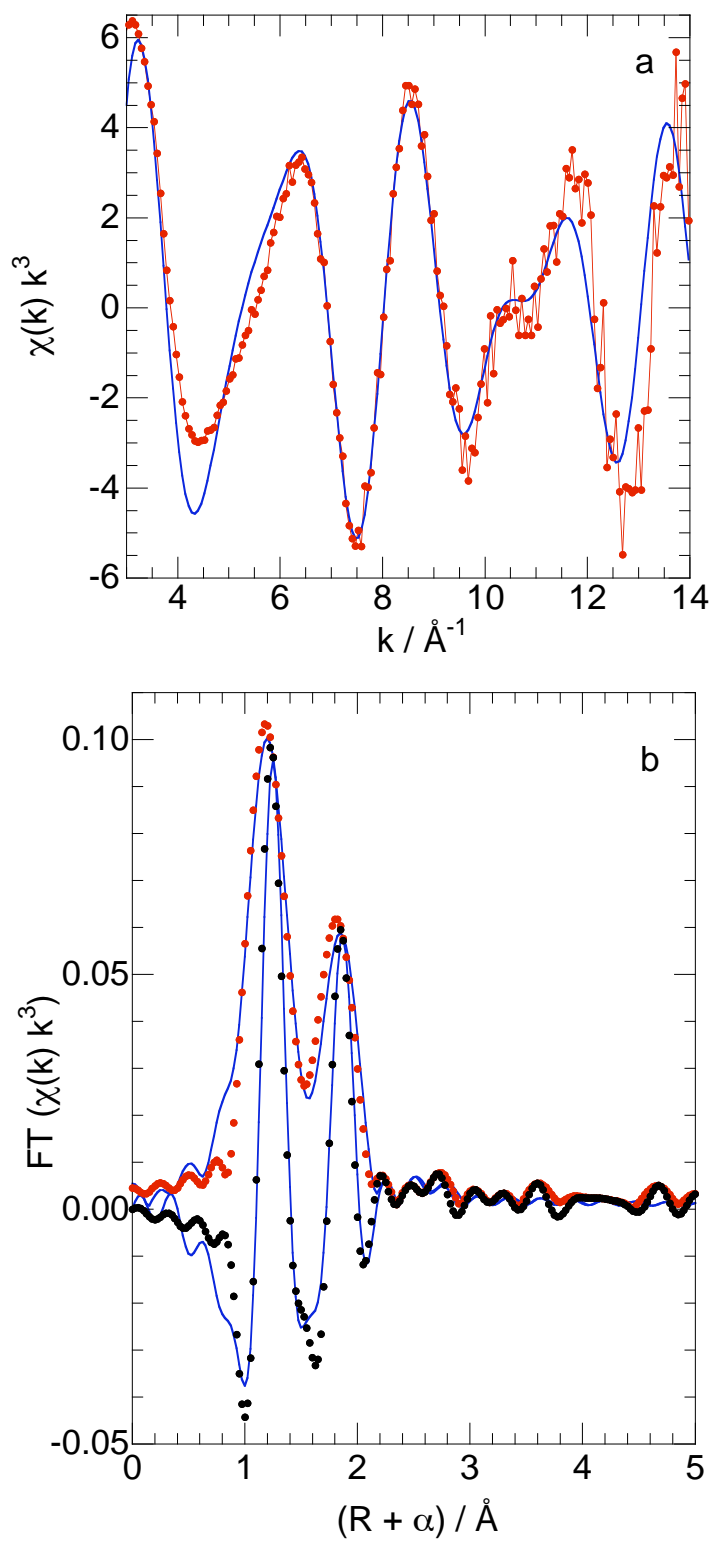
Table S1. Fit parameters for the single-scattering refinement ${ }^{a}$ of the $\equiv \mathrm{SiOCrO}_{2} \mathrm{Cl}$ model to EXAFS of $\mathrm{CrO}_{2} \mathrm{Cl}_{2}$ grafted on $\mathrm{S} 952-450$

\begin{tabular}{lcccc}
\hline path & $\mathrm{N}^{b}$ & $\mathrm{R}$ & $\sigma^{2}$ & $\Delta \mathrm{E}_{0}$ \\
& & $\AA$ & $\AA^{2}$ & $\mathrm{eV}$ \\
\hline $\mathrm{Cr}=\mathrm{O}$ & 2 & 1.60 & 0.0022 & 1.9 \\
$\mathrm{Cr}-\mathrm{O}$ & 1 & 1.79 & 0.0022 & 1.9 \\
$\mathrm{Cr}-\mathrm{Cl}$ & 1 & 2.14 & 0.0033 & -3.9 \\
\hline
\end{tabular}

${ }^{a}$ Residual $=14.0 ; \mathrm{S}_{0}{ }^{2}=0.98$. Errors for single-scattering paths representing atoms in the first coordination sphere of the metal, in the absence of systematic fitting errors, are generally accepted to be as follows: bond lengths $\pm 0.02 \AA, \sigma^{2} \pm 20 \%, \Delta \mathrm{E}_{0}, \pm 20 \% .{ }^{b}$ Coordination numbers were fixed at integer values. 
Figure S2. Single-scattering refinement of the $\equiv \mathrm{SiOCrO}_{2} \mathrm{Cl}$ model to EXAFS of $\mathrm{CrO}_{2} \mathrm{Cl}_{2}$ modified S952-800: (a) in $\mathrm{k}^{3}$-weighted k-space (red points and line); and (b) in non-phasecorrected R-space (imaginary, black points; FT magnitude, red points). Parameters for the curvefit (blue lines) are given in Table S2.
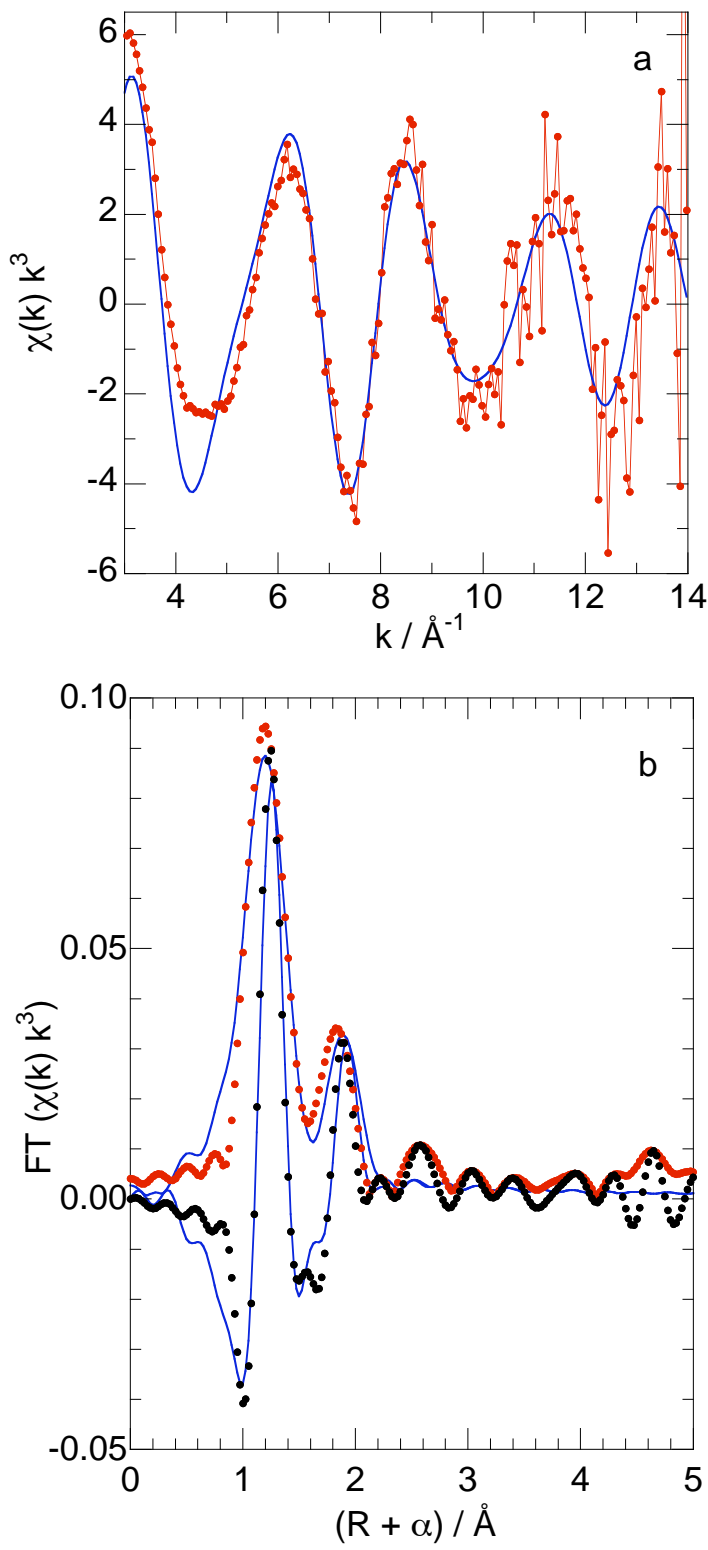
Table S2. Fit parameters for the single-scattering refinement ${ }^{a}$ of the $\equiv \mathrm{SiOCrO}_{2} \mathrm{Cl}$ model to EXAFS of $\mathrm{CrO}_{2} \mathrm{Cl}_{2}$ grafted on $\mathrm{S} 952-800$

\begin{tabular}{lcccc}
\hline path & $\mathrm{N}^{b}$ & $\mathrm{R}$ & $\sigma^{2}$ & $\Delta \mathrm{E}_{0}$ \\
& & $\AA$ & $\AA^{2}$ & $\mathrm{eV}$ \\
& & & & \\
\hline $\mathrm{Cr}=\mathrm{O}$ & 2 & 1.60 & 0.0031 & -0.8 \\
$\mathrm{Cr}-\mathrm{O}$ & 1 & 1.79 & 0.0050 & -0.8 \\
$\mathrm{Cr}-\mathrm{Cl}$ & 1 & 2.18 & 0.0078 & -3.4 \\
\hline
\end{tabular}

${ }^{a}$ Residual $=7.9 ; \mathrm{S}_{0}{ }^{2}=0.98$. Errors for single-scattering paths representing atoms in the first coordination sphere of the metal, in the absence of systematic fitting errors, are generally accepted to be as follows: bond lengths $\pm 0.02 \AA, \sigma^{2} \pm 20 \%, \Delta \mathrm{E}_{0}, \pm 20 \%$. ${ }^{b}$ Coordination numbers were fixed at integer values. 
Table S3. Cartesian Coordinates for Optimized Structure of I Absolute $E=-1174.66762270$ Hartrees $(B 3 L Y P / 6-311+G(2 d, p))$

$x$

$\mathrm{Si}$

$\mathrm{H}$

$\mathrm{H}$

$\mathrm{H}$

$\mathrm{Si}$

$\mathrm{H}$

$\mathrm{Si}$

$\mathrm{H}$

$\mathrm{H}$

$\mathrm{O}$

$\mathrm{O}$

$\mathrm{O}$

$\mathrm{O}$

$\mathrm{H}$

$\mathrm{H}$
$-0.1182062917$

0.8341481638

$-0.2213800202$

1.1587966766

$-0.0049975948$

$-1.1749220231$

0.1233886469

$-0.8288615148$

0.2362348608

0.4393121769

$-0.442485931$

1.6488316759

$-1.646150275$

$-1.7734958082$

1.7708318448 y

2.346721737

3.4743774412

1.681168057

$-0.3611624782$

$-0.0043220429$

0.3472168818

$-2.3422399087$

$-3.4698054171$

$-1.6683212254$

1.2844237132

$-1.2871684539$

$-2.8704644062$

2.8727833477

3.4931665974

$-3.4954704585$ $z$

$-0.6923071505$

$-0.6992405041$

$-2.000179276$

2.1793440008

1.3488757162

2.1729673315

$-0.7064363246$

$-0.7276523913$

$-2.0092161817$

0.4340594785

0.4225525612

$-0.364958966$

$-0.3587644898$

0.3641390381

0.354877936 
Table S4. Cartesian Coordinates for Optimized Structure of Ia

Absolute $\mathrm{E}=-1871.14803626$ Hartrees (B3LYP/LANL2DZ(for Cr); B3LYP/6-311+G(2d,p) for $\mathrm{O}, \mathrm{Si}, \mathrm{Cl}, \mathrm{H})$.

\begin{tabular}{|c|c|c|c|}
\hline & $x$ & $y$ & $z$ \\
\hline $\mathrm{Si}$ & 2.7536547377 & -1.6588627189 & -0.2508106217 \\
\hline $\mathrm{H}$ & 2.7388873342 & -2.2191800190 & -1.6148926542 \\
\hline $\mathrm{H}$ & 4.0402489089 & -1.8621507649 & 0.4357374634 \\
\hline $\mathrm{H}$ & 2.8485631741 & 1.1581004125 & 1.8036603681 \\
\hline $\mathrm{Si}$ & 2.4842692524 & 1.3696234224 & 0.3929320853 \\
\hline $\mathrm{H}$ & 3.3965684475 & 2.3075146491 & -0.2856632902 \\
\hline $\mathrm{Si}$ & -0.2611812883 & 2.4238099749 & -0.6080605192 \\
\hline $\mathrm{H}$ & -0.9549127885 & 3.5976354128 & -0.0581995575 \\
\hline $\mathrm{H}$ & 0.1815449898 & 2.5999382377 & -2.0021780285 \\
\hline $\mathrm{O}$ & 2.4766947519 & -0.0388451219 & -0.4398402433 \\
\hline $\mathrm{O}$ & 0.9685827543 & 2.0280774430 & 0.3790731175 \\
\hline $\mathrm{O}$ & -1.3990424551 & 1.1915671087 & -0.6796200665 \\
\hline $\mathrm{O}$ & 1.6332711892 & -2.3717185445 & 0.7133140005 \\
\hline $\mathrm{Cr}$ & -1.9854388372 & -0.1502453015 & 0.2233434323 \\
\hline $\mathrm{O}$ & -3.5324695495 & -0.0593813844 & 0.3700817355 \\
\hline $\mathrm{O}$ & -1.3365781669 & -0.1891681520 & 1.6360489236 \\
\hline $\mathrm{H}$ & 0.7261647434 & -2.4480954150 & 0.3954916698 \\
\hline $\mathrm{Cl}$ & -1.4990940431 & -1.9938891509 & -0.7849124297 \\
\hline
\end{tabular}


Table S5. Cartesian Coordinates for Optimized Structure of Ib

Absolute E = -1871.14576219 Hartrees (B3LYP/LANL2DZ(for Cr); B3LYP/6-311+G(2d,p) for $\mathrm{O}, \mathrm{Si}, \mathrm{Cl}, \mathrm{H})$.

$y \quad z$

\begin{tabular}{|c|c|c|c|}
\hline $\mathrm{Si}$ & -2.9925857151 & 1.5868072636 & 0.1171016053 \\
\hline $\mathrm{H}$ & -2.4298454031 & 1.8393846909 & 1.4614830295 \\
\hline $\mathrm{H}$ & -2.2420289858 & 2.2448124292 & -0.9588918376 \\
\hline $\mathrm{H}$ & -2.4728099962 & -1.3090725916 & 2.0141091770 \\
\hline $\mathrm{Si}$ & -2.6269748043 & -1.4805543648 & 0.5568951033 \\
\hline $\mathrm{H}$ & -3.6974985082 & -2.4317530885 & 0.2209363376 \\
\hline $\mathrm{Si}$ & 0.0500704935 & -1.6572530633 & -0.9702406528 \\
\hline $\mathrm{H}$ & 1.0124370158 & -2.7689627704 & -0.9998751725 \\
\hline $\mathrm{H}$ & -0.3847440850 & -1.2119898667 & -2.3042462953 \\
\hline $\mathrm{O}$ & -2.9570771786 & -0.0410994856 & -0.1491833987 \\
\hline $\mathrm{O}$ & -1.2171194668 & -2.0913204337 & -0.0433647995 \\
\hline $\mathrm{O}$ & 0.8029932453 & -0.3263326849 & -0.2763301943 \\
\hline $\mathrm{O}$ & -4.5242638344 & 2.1774309817 & 0.0090009434 \\
\hline $\mathrm{Cr}$ & 2.3683704829 & 0.1053729702 & 0.3072772482 \\
\hline $\mathrm{O}$ & 3.2833418431 & -1.1552529909 & 0.3239093154 \\
\hline $\mathrm{O}$ & 2.2535461053 & 0.6772874532 & 1.7515167614 \\
\hline $\mathrm{H}$ & -5.1372442548 & 2.0248330622 & 0.7330065325 \\
\hline $\mathrm{Cl}$ & 3.2560198400 & 1.5807115551 & -0.9598911487 \\
\hline
\end{tabular}


Table S6. Cartesian Coordinates for Optimized Structure of Ic

Absolute E = -1871.14960695 Hartrees (B3LYP/LANL2DZ(for Cr); B3LYP/6-311+G(2d,p) for $\mathrm{O}, \mathrm{Si}, \mathrm{Cl}, \mathrm{H})$.

\begin{tabular}{|c|c|c|c|}
\hline & $x$ & $y$ & $z$ \\
\hline $\mathrm{Si}$ & 2.6499204786 & -1.7290713699 & 0.3881274333 \\
\hline $\mathrm{H}$ & 2.1524311885 & -2.6278067893 & -0.6697571729 \\
\hline $\mathrm{H}$ & 4.0348699117 & -2.0332464652 & 0.7829231421 \\
\hline $\mathrm{H}$ & 3.4057407591 & 1.5779008243 & 1.3885680582 \\
\hline $\mathrm{Si}$ & 2.5582779779 & 1.3576544336 & 0.2049191663 \\
\hline $\mathrm{H}$ & 2.9739194112 & 2.1790280352 & -0.9465338813 \\
\hline $\mathrm{Si}$ & -0.3476680863 & 2.4874626066 & 0.0928944710 \\
\hline $\mathrm{H}$ & -0.9523545888 & 3.2766151867 & 1.1756717727 \\
\hline $\mathrm{H}$ & -0.1242643960 & 3.2546778536 & -1.1454278120 \\
\hline $\mathrm{O}$ & 2.5853199119 & -0.2062565795 & -0.2591993756 \\
\hline $\mathrm{O}$ & 1.0221220714 & 1.8027606731 & 0.6341322451 \\
\hline $\mathrm{O}$ & -1.4844871915 & 1.3277570564 & -0.3299049501 \\
\hline $\mathrm{O}$ & 1.7655645526 & -1.8459151021 & 1.7668238759 \\
\hline $\mathrm{Cr}$ & -1.9352402888 & -0.3180514568 & -0.1245623470 \\
\hline $\mathrm{O}$ & -3.4184021483 & -0.4011845420 & 0.3380062791 \\
\hline $\mathrm{O}$ & -1.0237203019 & -1.0204009641 & 0.9349698091 \\
\hline $\mathrm{H}$ & 0.8082316554 & -1.7421888852 & 1.7001952465 \\
\hline $\mathrm{Cl}$ & -1.7336121969 & -1.3606157951 & -1.9751730048 \\
\hline
\end{tabular}


Table S7. Cartesian Coordinates for Optimized Structure of II Absolute $\mathrm{E}=-808.597241256$ Hartrees $(\mathrm{B} 3 \mathrm{LYP} / 6-311+\mathrm{G}(2 \mathrm{~d}, \mathrm{p}))$

$x$

$\mathrm{Si}$

$\mathrm{H}$

$\mathrm{H}$

$\mathrm{H}$

$\mathrm{Si}$

$\mathrm{H}$

$\mathrm{O}$

$\mathrm{O}$

$\mathrm{O}$

$\mathrm{H}$

$\mathrm{H}$
1.4566433183

2.4218211375

1.4496792216

$-2.4306671341$

$-1.5663389617$

$-1.9690382553$

$-0.0190733610$

$-1.6963950787$

1.8568785719

2.1848451954

$-1.2521822157$

$$
y
$$

$-0.3930942474$

$-1.4322317171$

$-0.1567307289$

$-1.2512385856$

$-0.3341231902$

$-0.2309195931$

$-0.9021578940$

1.1218237265

1.0668713765

1.0752470065

1.8846200730 $z$

$-0.3016256400$

0.1059253004

$-1.7534598376$

$-0.5260516872$

0.2311549307

1.6491048057

0.2115937923

$-0.5313952925$

0.3684182713

1.2717922889

$-0.1496551096$ 
Table S8. Cartesian Coordinates for Optimized Structure of IIa Absolute $\mathrm{E}=-1505.07638784$ Hartrees (B3LYP/LANL2DZ(for Cr); B3LYP/6-311+G(2d,p) for $\mathrm{O}, \mathrm{Si}, \mathrm{Cl}, \mathrm{H})$.

\begin{tabular}{|c|c|c|c|}
\hline & $x$ & $y$ & $z$ \\
\hline $\mathrm{H}$ & -4.0473052147 & 0.3565499467 & 1.1790308003 \\
\hline $\mathrm{Si}$ & -2.8857425180 & 0.6886875860 & 0.3405436827 \\
\hline $\mathrm{H}$ & -3.2595538344 & 1.3383702024 & -0.9280604376 \\
\hline $\mathrm{Si}$ & -1.0244897309 & -1.7889333865 & 0.4726934488 \\
\hline $\mathrm{H}$ & -1.3642661575 & -2.2545484812 & 1.8271092495 \\
\hline $\mathrm{H}$ & -0.8786049460 & -2.8730944019 & -0.5096349213 \\
\hline $\mathrm{O}$ & -1.9317130220 & 1.6454707501 & 1.2740201644 \\
\hline $\mathrm{O}$ & -2.1050543909 & -0.7103866331 & -0.0893474710 \\
\hline $\mathrm{O}$ & 0.4918896903 & -1.0686669459 & 0.6391222017 \\
\hline $\mathrm{Cr}$ & 1.5489351898 & -0.2167378233 & -0.4180840650 \\
\hline $\mathrm{O}$ & 1.1932441291 & -0.5393324673 & -1.8993803983 \\
\hline $\mathrm{O}$ & 3.0231574910 & -0.6294671201 & -0.1392134363 \\
\hline $\mathrm{Cl}$ & 1.3454718039 & 1.9081064146 & -0.1235304141 \\
\hline $\mathrm{H}$ & -1.1266747649 & 2.0191219834 & 0.8986615824 \\
\hline
\end{tabular}


Table S9. Cartesian Coordinates for Optimized Structure of IIb

Absolute $\mathrm{E}=-1505.07511448$ Hartrees (B3LYP/LANL2DZ(for Cr); B3LYP/6-311+G(2d,p) for $\mathrm{O}, \mathrm{Si}, \mathrm{Cl}, \mathrm{H})$.

\begin{tabular}{|c|c|c|c|}
\hline & $x$ & $y$ & $z$ \\
\hline $\mathrm{H}$ & 2.7330667562 & 1.7049961056 & 0.9791721740 \\
\hline $\mathrm{Si}$ & 3.0362613754 & 0.6493198240 & -0.0028452372 \\
\hline $\mathrm{H}$ & 3.1589408732 & 1.1467471610 & -1.3822457211 \\
\hline $\mathrm{Si}$ & 0.8607259997 & -1.4182044287 & -0.8208835265 \\
\hline $\mathrm{H}$ & 1.3406616934 & -1.4756409499 & -2.2126557546 \\
\hline $\mathrm{H}$ & 0.7663997262 & -2.7358537243 & -0.1737876651 \\
\hline $\mathrm{O}$ & 4.4754883661 & -0.0735985215 & 0.3289414020 \\
\hline $\mathrm{O}$ & 1.7893573015 & -0.4326848409 & 0.0854476514 \\
\hline $\mathrm{O}$ & -0.6961662446 & -0.8066757959 & -0.9223886120 \\
\hline $\mathrm{Cr}$ & -1.8205986898 & -0.1064552861 & 0.1876293085 \\
\hline $\mathrm{O}$ & -1.4514010895 & -0.4978576299 & 1.6494276673 \\
\hline $\mathrm{O}$ & -3.2550875902 & -0.6184931326 & -0.1337873557 \\
\hline $\mathrm{Cl}$ & -1.7899654504 & 2.0262921106 & 0.0308055994 \\
\hline $\mathrm{H}$ & 4.6693629643 & -0.3334237754 & 1.2337950407 \\
\hline
\end{tabular}


Table S10. Cartesian Coordinates for Optimized Structure of IIc Absolute $\mathrm{E}=-1505.07754043$ Hartrees (B3LYP/LANL2DZ(for Cr); B3LYP/6-311+G(2d,p) for $\mathrm{O}, \mathrm{Si}, \mathrm{Cl}, \mathrm{H})$.

$x \quad y \quad z$

$\begin{array}{llll}\mathrm{H} & 3.6701801473 & 1.8710542148 & 0.1202710601 \\ \mathrm{Si} & 2.3827841216 & 1.6950800186 & 0.8102209505 \\ \mathrm{H} & 2.3563861223 & 2.3120132972 & 2.1480110637 \\ \mathrm{Si} & 1.7421268390 & -1.3153091312 & 0.3248453200 \\ \mathrm{H} & 2.4954195653 & -1.4962168918 & -0.9283912318 \\ \mathrm{H} & 1.9168718246 & -2.4199587838 & 1.2781933011 \\ \mathrm{O} & 1.2571787457 & 2.3368342741 & -0.1982508462 \\ \mathrm{O} & 2.1166888221 & 0.0787301170 & 1.0712301853 \\ \mathrm{O} & 0.1267752828 & -1.3417121857 & -0.1343063488 \\ \mathrm{Cr} & -1.3676908992 & -0.4997966845 & -0.0536792008 \\ \mathrm{O} & -1.2126542653 & 0.8202047925 & 0.7721461495 \\ \mathrm{O} & -2.4465327264 & -1.3989730491 & 0.6168088317 \\ \mathrm{Cl} & -2.0254289184 & 0.0067756005 & -2.0196644815 \\ \mathrm{H} & 0.3376152135 & 2.3355793717 & 0.0925612513\end{array}$


Table S11. Cartesian Coordinates for Optimized Structure of III Absolute $\mathrm{E}=-883.910338697$ Hartrees; B3LYP/6-311+G(2d,p) for O, Si, H).

$x$

$\mathrm{Si}$

$\mathrm{H}$

$\mathrm{Si}$

$\mathrm{O}$

$\mathrm{O}$

$\mathrm{H}$

$\mathrm{H}$

$\mathrm{O}$

$\mathrm{H}$

$\mathrm{H}$

$\mathrm{O}$

$\mathrm{H}$
$-1.7826645211$

$-2.5559315784$

1.2821855089

$-0.2118578259$

1.2499630761

1.2008792436

1.9611786896

2.0768966961

1.8136203134

$-2.1987716159$

$-2.0239708251$

$-1.9425178511$ $y$

$-0.349756171$

$-1.1960508879$

0.1262431282

$-0.50125348$

1.6554572996

2.380052181

$-0.6891372468$

0.1485339757

$-0.4945148082$

1.06072955

$-0.8246320159$

$-1.756742424$ $z$

$-0.0776631963$

0.8526557591

0.1236031297

0.3916066123

$-0.457496675$

0.1726581957

$-0.8859810404$

1.5632406409

2.2270019617

$-0.039621384$

$-1.6366177575$

$-1.8557351251$ 
Table S12. Cartesian Coordinates for Optimized Structure of IV

Absolute $\mathrm{E}=-1119.53584883$ Hartrees (B3LYP/LANL2DZ(for Cr); B3LYP/6-311+G(2d,p) for $\mathrm{O}, \mathrm{Si}, \mathrm{Cl}, \mathrm{H})$.

$\begin{array}{ll}x & y\end{array}$

$\begin{array}{llrl}\mathrm{Si} & -0.4784139792 & 2.003477017 & 0.5989140271 \\ \mathrm{H} & -0.2617312045 & 3.3457878371 & 0.0391399929 \\ \mathrm{H} & -1.0274307747 & 2.0304104036 & 1.9643303736 \\ \mathrm{Si} & -1.3910716174 & -0.2687530666 & -1.2326651735 \\ \mathrm{H} & -1.6807517129 & -0.0510326174 & -2.6453504262 \\ \mathrm{O} & -1.4795516728 & 1.1373796591 & -0.3828655833 \\ \mathrm{O} & 0.1948671556 & -0.7854894443 & -1.0814984610 \\ \mathrm{O} & 1.0031723625 & 1.2219868065 & 0.6112148132 \\ \mathrm{Cr} & 1.1625081944 & -0.4961718500 & 0.3402559612 \\ \mathrm{O} & 2.6486501125 & -0.9177968518 & 0.1359134539 \\ \mathrm{O} & 0.5310633158 & -1.2712043334 & 1.5500330333 \\ \mathrm{O} & -2.4444800717 & -1.3844970365 & -0.6910154408 \\ \mathrm{H} & -2.3872542371 & -1.7062069261 & 0.2139985173\end{array}$


Table S13. Cartesian Coordinates for Optimized Structure of V

Absolute $\mathrm{E}=-807.403827702$ Hartrees; B3LYP/6-311+G(2d,p) for O, Si, H).

$x$

$\mathrm{Si}$

$\mathrm{H}$

$\mathrm{Si}$

$\mathrm{H}$

$\mathrm{H}$

$\mathrm{O}$

$\mathrm{O}$

$\mathrm{O}$

$\mathrm{H}$
$-0.8871350541$

$-2.0432602851$

1.3929087088

1.8148582228

2.5470404219

0.1356307647

0.3615809314

$-1.3822137582$

$-2.3194530282$ $y$

$-0.0528808313$

$-0.5000121892$

$-0.1161219407$

$-0.6192942270$

0.3345482995

1.0033435823

$-1.1682612528$

0.4978308327

0.4874916274 $z$

$-0.0950492630$

0.6929470460

0.6293861564

1.9483726695

$-0.1676483374$

0.7115273174

$-0.1891878777$

$-1.5469851147$

$-1.7572224856$ 
Table S14. Cartesian Coordinates for Optimized Structure of VI Absolute $\mathrm{E}=-1503.90619175$ Hartrees (B3LYP/LANL2DZ(for Cr); B3LYP/6-311+G(2d,p) for $\mathrm{O}, \mathrm{Si}, \mathrm{Cl}, \mathrm{H})$.

$\begin{array}{lll}x & y\end{array}$

$\begin{array}{llll}\mathrm{Si} & -1.1856679799 & 1.8785180465 & -0.2700816650 \\ \mathrm{H} & -1.8614916007 & 2.6150807456 & -1.3475768070 \\ \mathrm{H} & -1.2891989455 & 2.5355553945 & 1.0413765375 \\ \mathrm{Si} & -1.1889626928 & -1.1628634394 & -0.2757429081 \\ \mathrm{H} & -1.8678106171 & -1.9463197098 & -1.3045320317 \\ \mathrm{O} & -1.8072034470 & 0.3486585485 & -0.1794568457 \\ \mathrm{O} & 0.4153829286 & -1.0292155450 & -0.6725068035 \\ \mathrm{O} & 0.4245643093 & 1.7247076347 & -0.7027084105 \\ \mathrm{Cr} & 1.4162487192 & 0.3553811819 & -0.2644291988 \\ \mathrm{O} & 2.7689916665 & 0.3439871317 & -1.0364572774 \\ \mathrm{O} & 1.6759607291 & 0.3736743001 & 1.2769924939 \\ \mathrm{Cl} & -1.3851887159 & -2.1086544621 & 1.5359806965\end{array}$


Table S15. Cartesian Coordinates for Optimized Structure of $\mathrm{CrO}_{2} \mathrm{Cl}_{2}$

Absolute $\mathrm{E}=-1157.31508795$ Hartrees (B3LYP/LANL2DZ(for Cr); B3LYP/6-311+G(2d,p) for $\mathrm{O}, \mathrm{Cl})$.

$\begin{array}{cccc} & x & y & z \\ \mathrm{Cr} & 0.289433179 & 0.0000000000 & 0.2046601636 \\ \mathrm{O} & 0.298485845 & 0.0000000000 & 1.7565867640 \\ \mathrm{O} & 1.755621165 & 0.0000000000 & -0.3041137678 \\ \mathrm{Cl} & -0.687625070 & -1.7493446422 & -0.4862243499 \\ \mathrm{Cl} & -0.687625070 & 1.7493446422 & -0.4862243499\end{array}$


Table S16. Cartesian Coordinates for Optimized Structure of $\mathrm{HCl}$ Absolute $E=-460.835445494$ Hartrees $(B 3 L Y P / 6-311+G(2 d, p))$.

$\begin{array}{cccc} & x & y & z \\ \mathrm{Cl} & 0.0000000000 & 0.0000000000 & -0.0712500064 \\ \mathrm{H} & 0.0000000000 & 0.00000000000 & 1.2112501093\end{array}$

Table S17. Cartesian Coordinates for Optimized Structure of $\mathrm{H}_{2} \mathrm{O}$ Absolute $E=-76.4595257424$ Hartrees $(B 3 L Y P / 6-311+G(2 d, p))$.

$x$

$\mathrm{O}$

$\mathrm{H}$

$\mathrm{H}$ $y$

$-0.0674932628$

0.8948309333

$-0.3548848312$
$-0.0955007584$

$-0.0596028548$

0.8236089218 $z$

0.0000000000

0.0000000000

0.0000000000 